\title{
The auditory evoked sustained field: origin and frequency dependence
}

\author{
C. Pantev *, C. Eulitz, T. Elbert and M. Hoke \\ Wilhelms-University of Münster, Institute of Experimental Audiology, Centre of Biomagnetism, Kardinal-von-Galen-Ring 10, \\ D-48129 Münster (Germany)
}

\begin{abstract}
Summary A sound lasting for several seconds is known to elicit a basline shift in electrical and magnetic records. We have studied the dependence of the magnetic field distribution of this "per-stimulatory" sustained field (SF) on tone frequency. Tone bursts of 2 sec duration and $60 \mathrm{~dB} \mathrm{nHL}$ intensity were presented to 11 subjects at varying interstimulus intervals between 5 and 7 sec. The carrier frequencies of 250 , 1000 and $4000 \mathrm{~Hz}$ varied randomly from trial to trial. The field distributions obtained are consistent with the view that the auditory evoked sustained field activity originates in the supratemporal cortex. Differences in the locations of equivalent current dipoles of the SF from those of the M100 wave of the slow auditory evoked field are consistent across subjects. The SF source locations corresponding to stimulus frequencies over an extended frequency range are arranged in a tonotopic manner and support the idea that the sources of the M100 and the SF are current dipole sheets located on the superior surface of the primary auditory cortex.
\end{abstract}

Key words: Auditory evoked sustained field; Auditory evoked field; Auditory evoked sustained potential; Auditory evoked potential; EEG; MEG

A transient auditory stimulus elicits several transient deflections (auditory evoked responses) following the onset of the stimulus (Picton et al. 1974). If the stimulus is of longer duration, the transient deflections of the on-effect are followed by a stimulus-locked DC shift that lasts as long as the stimulus is present. This "per-stimulatory" DC response is called sustained potential (SP) in electrical records, or sustained field (SF) in magnetic records. It is usually followed by an off-response which is time-locked to the end of the transient auditory stimulus. Although evidence for the SP was provided by Köhler et al. as early as 1952 , using DC recording from the auditory cortex of the cat, and by Köhler and Wegener in 1955 in DC scalp records of humans, the number of investigations of the SP and SF during the following 40 years has remained small compared to the number of studies of transient slow auditory evoked potentials (AEP) and fields (AEF). One reason for this difference may be technical difficulties, as electrical records are contaminated by electrode drifts and skin potentials. Such artifacts do not appear in magnetic records. Experimental studies of auditory evoked sustained potentials include those by David et al. (1969), Keidel (1971), Järvilehto and Fruhstorfer (1973), Picton et al. (1977, 1978a,b) and Hari et al.

\footnotetext{
* Corresponding author.
}

(1979a,b). Sustained fields were investigated for the first time by Hari (1980) and Hari et al. (1980, 1987). Across the scalp, the auditory evoked sustained negative potential shift becomes largest over the frontocentral regions. This is probably a consequence of volume conducted activity generated in the supratemporal cortex. With respect to functional behaviour, the auditory sustained potential is quite distinct from the slow transient AEP that occurs at the onset and offset of auditory stimuli. The excellent experimental study of Picton et al. (1978a) revealed that, whereas the transient AEP can be attenuated by intermixing a series of clicks and tonebursts, this manipulation leaves the SP unchanged. This means that the two phenomena, the slow AEP and the SP, have different refractory properties as well as generator mechanisms and, thus, physiologically distinct generators.

The objection of Järvilehto and Fruhstorfer (1973) that the SP is probably a type of contingent negative variation (CNV), associated with subjective uncertainty about the timing of stimulus onset (rather than a sensory response to the auditory stimulus), has been disproved by the results of Picton et al. (1978a) which show that, in contrast to the CNV, an SP can be recorded even in the absence of any attention to the stimulus. The auditory SP can be recorded during the waking state as well as during sleep, as opposed to the $\mathrm{CNV}$, which requires a sufficient degree of attentive 
expectancy. Therefore it seems that the auditory SP and $\mathrm{CNV}$ are independently generated phenomena that can be concurrently recorded if an appropriate stimulus paradigm is used (Picton et al. 1978a). This view receives support from the localization results for the SP reported by Scherg et al. (1989) and Scherg and Picton (1991), and the localization of the SF reported by Hari (1980) and Hari et al. (1980, 1987). These authors found the sources of the SP and the SF to be located in the supratemporal cortex near the sources of the major component of the slow AEF (M100), having a latency of about $100 \mathrm{msec}$ after the stimulus onset, which has been repeatedly shown to indicate the location of the auditory cortex and association areas (Yamamoto et al. 1988; Pantev et al. 1990; Papanicolaou et al. 1990; Pantev 1992). On the other hand, the magnetic counterpart of the CNV, the contingent magnetic variation (CMV), cannot be accounted for by a single equivalent dipole (Elbert et al. 1993).

Tonotopicity is one of the general principles of the functional organization of the auditory system. That tonotopic organization is maintained throughout the central auditory pathway, including the primary auditory cortex, is known not only from animal studies (Merzenich and Brugge 1973; Merzenich et al. 1975, 1976; Schreiner 1991) but also from human studies (Elberling et al. 1982; Romani et al. 1982; Lauter et al. 1985; Pantev et al. 1988, 1989, 1991a; Yamamoto et al. 1988; Bertrand et al. 1991). The depth of the source of the M100 component of the slow AEF indicates the tonotopic organization of the auditory cortex: the higher the stimulus frequency, the deeper the equivalent source of the M100 component. Moreover, in most subjects the depth of the M100 equivalent source increases linearly with the logarithm of the stimulus frequency.

Existing information concerning the origin of the auditory evoked sustained response is still insufficient: there exists practically no information about the behaviour of the source of the sustained activity as the stimulus frequency changes. Picton et al. (1978b), using the technique of Butler (1968), investigated the frequency specificity of the refractory period of the SP to gain further knowledge of the physiological characteristics of the sustained potential generator. Their results were easily interpreted in terms of overlapping receptive fields for the generator processes at each frequency. Whereas the $\mathrm{N} 100$ component of the slow AEP has a well-defined receptive field, the expression of frequency specificity in the SP was much more complex. The results suggested that there might be two distinct generator processes for the auditory SP, one non-specific in its frequency receptive field, and one specific (Picton et al. 1978b).

This paper reports the results of a neuromagnetic study designed to investigate the nature and the sources of the auditory SF as well as their tonotopic organization, compared with the corresponding tonotopic organization of the sources of the M100 component of the slow AEF. Based on our experimental findings it appears that the auditory evoked sustained activity, or at least the part generated by the tangentially oriented sources which can be recorded magnetically, is indeed an auditory sensory response originating in the supratemporal cortex. The sources of the SF are quite distinct from those of the slow AEF. The source locations corresponding to stimulus frequencies over an extended frequency range differ in space. These results support the idea that the sources of the M100 and the SF are current dipole sheets located at the superior surface of the primary auditory cortex (Hari 1980).

\section{Methods}

\section{(a) Subjects}

Six male and 5 female subjects with no history of otological or neurological disorders and normal audiological status (air conduction and bone conduction thresholds in the range from 250 to $8000 \mathrm{~Hz}$ no more than $10 \mathrm{~dB}$ hearing level), aged between 21 and 33 years (mean age 25.8 years), participated in this study. All subjects were right-handed as determined with a modified handedness questionnaire of Annett (1967). Informed consent was obtained from each subject after the nature of the study was fully explained to her or him. The subjects were paid for their participation.

\section{(b) Stimulation}

Stimuli were delivered to the right ear, and AEFs were recorded from the left hemisphere, since previous studies have shown that stronger AEFs are recorded over the hemisphere contralateral to the side of handedness (Elberling et al. 1981; Hoke 1988), and contralateral to the side of stimulation (Elberling et al. 1981; Reite et al. 1981 Pantev et al. 1986). Tone bursts of $2 \mathrm{sec}$ duration (10 msec rise and decay time, cosine function) and $60 \mathrm{~dB} \mathrm{nHL}$ (normative hearing level) were presented to the subject's right ear (contralateral to the investigated hemisphere) with an interstimulus interval randomized between 5 and $7 \mathrm{sec}$. The carrier frequencies of 250,1000 and $4000 \mathrm{~Hz}$ were varied randomly, and each frequency appeared a total of 128 times within the 2 blocks of 192 stimuli each. The stimuli were presented through a non-magnetic and echo-free stimulus delivery system with an almost linear frequency characteristic (deviations less than \pm 4 $\mathrm{dB}$ in the range between 200 and $4000 \mathrm{~Hz}$ ). During stimulus presentation the subjects were asked to keep their eyes open, to stay awake, and to minimize eye movements and eye blinks during stimulus presentations. 


\section{(c) Neuromagnetic recording}

Recording was carried out in a magnetically shielded room (Vacuumschmelze) using a 37-channel biomagnetometer (Magnes ${ }^{\mathrm{TM}}$, Biomagnetic Technologies). The detection coils of the biomagnetometer are arranged in a circular concave array $144 \mathrm{~mm}$ in diameter, with a spherical radius of $122 \mathrm{~mm}$. The axes of the detection coils are normal to the surface of the sensor array. The distance between the centres of two adjacent coils is 22 $\mathrm{mm}$; each coil itself measures $20 \mathrm{~mm}$ in diameter. The sensors are configurated as first-order axial gradiometers with a baseline of $50 \mathrm{~mm}$. Each coil is connected to a superconducting quantum interference device (SQUID) that produces a voltage proportional to the field radial to the coil. The spectral density of the intrinsic noise of each channel was between 5 and 7 $\mathrm{fT} / \sqrt{\mathrm{Hz}}$. The subjects were in a right lateral position with their head and neck supported by a specially fabricated vacuum cushion. A sensor position indicator system determined the spatial locations of the sensors relative to the head and also indicated if head movements occurred during the recording. The sensor array was centred over a point lying about $1.5 \mathrm{~cm}$ superior to the position T3 of the 10-20 system for electrode placement, as near as possible to the subjects head. Using a bandwidth from DC to $100 \mathrm{~Hz}$, stimulus-related epochs of $2400 \mathrm{msec}$ (including $200 \mathrm{msec}$ prestimulus baseline) were recorded and stored for later analysis.

\section{(d) Data analysis}

Wide-band responses to each of the two blocks were first selectively averaged after artifact rejection, which discarded about $5-10 \%$ of artifact-contaminated epochs due to eye blinks or muscle activity. The peakto-peak amplitude threshold criterion was set to 3000 fT. Since the wave forms of the averaged responses recorded in the two blocks were reasonably similar for all subjects (test/retest criterion), the average response across both blocks was used for further evaluation. The wide-band responses were digitally filtered using a second-order zero phase-shift Butterworth filter $(12 \mathrm{~dB} / \mathrm{oct})$. For the analysis of the SF, the low-pass was set to $5 \mathrm{~Hz}$. For the analysis of the M100 component, responses were filtered using a low-pass of $20 \mathrm{~Hz}$. After subtracting the prestimulus baseline, root-meansquare (RMS) field values over the 37 recording channels were calculated for every sampling point.

Source analyses using a single moving dipole model were applied to both obtained field distributions: DC-5 $\mathrm{Hz}$ and $\mathrm{DC}-20 \mathrm{~Hz}$. A spherical model was fitted to the digitized head shape for each subject, and the location ( $x, y, z$ positions), orientation, and amplitude of a best-fitting equivalent current dipole (Sarvas 1987) were estimated for each time point. The origin of the headbased coordinate system (determined by the sensor position indicator system) was the midpoint of the line between the preauricular points. The $\mathrm{x}$-axis joined the origin to the nasion; the $y$-axis passed between the preauricular points with positive values towards the left preauricular point. The $\mathrm{z}$-axis was perpendicular to the $x-y$ plane. Correlations between the theoretical field generated by the model and the observed field were used to estimate the goodness of fit of the model parameters. The $\mathrm{x}, \mathrm{y}$ and $\mathrm{z}$ coordinates of the dipole location with their confidence volumes were calculated in the above described head frame-based Cartesian coordinate system. Only estimates with a goodness of fit above 0.90 were considered for further analysis.

The source locations of the M100 were estimated after the usual criteria for best matching of: the averaged M100 peak latency in the recording channels around the maximum and the minimum of the measured field distribution, the maximal RMS, the maximal dipole moment and the maximal goodness of fit in the time range of $\pm 10 \mathrm{msec}$ around the averaged M100 peak (Pantev et al. 1991a,b). The estimation of

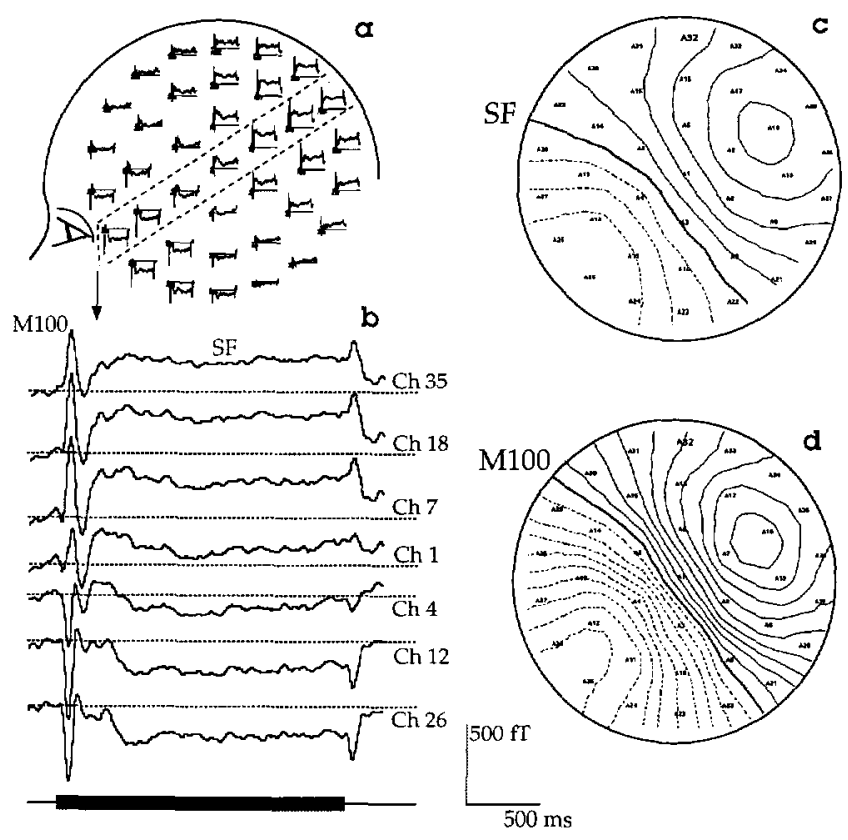

Fig. 1. a: set of 37 averaged wave forms of $2400 \mathrm{msec}$ length $(\mathrm{N}=128)$, obtained in the passband between $\mathrm{DC}$ and $100 \mathrm{~Hz}$ from the left hemisphere of one subject in response to a $2 \mathrm{sec}, 1000 \mathrm{~Hz}$ tone burst, schematically shown at the bottom of part $b$. The wave forms are projected onto a sketch of the head (not precisely in scale). $b$ : enlarged presentation of the magnetic wave forms in the region marked by dotted lines in a, connecting the minimum and the maximum of the sustained field pattern. Clear extrema and polarity reversal are evident for both the $\mathrm{M} 100$ of the slow AEF, in the onand off-responses and also for the sustained field. c: isofield contour map of the sustained field at a latency of $1300 \mathrm{msec}$ after stimulus onset. Outward going flux is marked by solid lines in the contour plot, inward going flux by dotted lines. Spacing between two adjacent isocontour lines is $50 \mathrm{fT}$. d: isofield contour map of the M100 at latency of $94 \mathrm{msec}$ after the stimulus onset. Plot parameters are the same as in $\mathrm{c}$. 
the equivalent source locations of the evoked sustained field proved to be more difficult. This assessment was based on the equivalent current dipole parameters in the time range of $800-2000 \mathrm{msec}$ after the stimulus onset, which was assumed to be free of overlapping transient event-related components. For the calculation of the averaged source location of the SF we used only those data points which had RMS values of at least 3 times higher than the average RMS during the prestimulus baseline. A second criterion for acceptance required that the goodness of fit of the data points within the range of $800-2000 \mathrm{msec}$ had to be greater than 0.9 . If these conditions were not met, the corresponding data points were excluded from further analysis. The averaged $x, y, z$ values of the equivalent current dipole in 3 subdivisions of $400 \mathrm{msec}$ of the SF range of $1200 \mathrm{msec}(800-2000 \mathrm{msec})$ were calculated for the remaining data points. If these values were similar they were averaged. Finally, these averaged values were taken to represent the space locations of the equivalent SF sources and so were submitted to further statistical evaluation using a multivariate analysis of variance (MANOVA) with the 3 different tone frequencies $(250,1000$ and $4000 \mathrm{~Hz})$, comprising one within-subject factor, and the two components (M100 and SF) another within-subject factor.

\section{Results}

Fig. 1a shows one representative set of data of one subject in response to the $1000 \mathrm{~Hz}$ tone burst. Traces are projected onto a sketch of the head. The well-defined dipolar structures of the SF and the slow AEF (on- and off-response) can be easily recognized (the results on the off-response will be presented in a separate paper).

Details of the wave forms appear in the enlarged presentation of the region marked by dotted lines in Fig. 1a. It shows magnetic activity in the supratemporal cortex recorded from channels located along the line connecting the minimum and the maximum of the field, a line which corresponds well with the course of the Sylvian fissure (Fig. 1b). Clear extrema and polarity reversals are evident for the pronounced M100 (at stimulus onset and offset) and also for the sustained field. Fig. 1c presents the isofield contour map of the sustained field at a latency of $1300 \mathrm{msec}$, Fig. 1d the isofield contour map of the M100 peak. Both field distributions resemble the theoretical field distribution of a current dipole, so they can be described in terms of the parameters of an equivalent current dipole. Inspection of the isocontour plots of the M100 and the SF demonstrates not only a difference in density of the
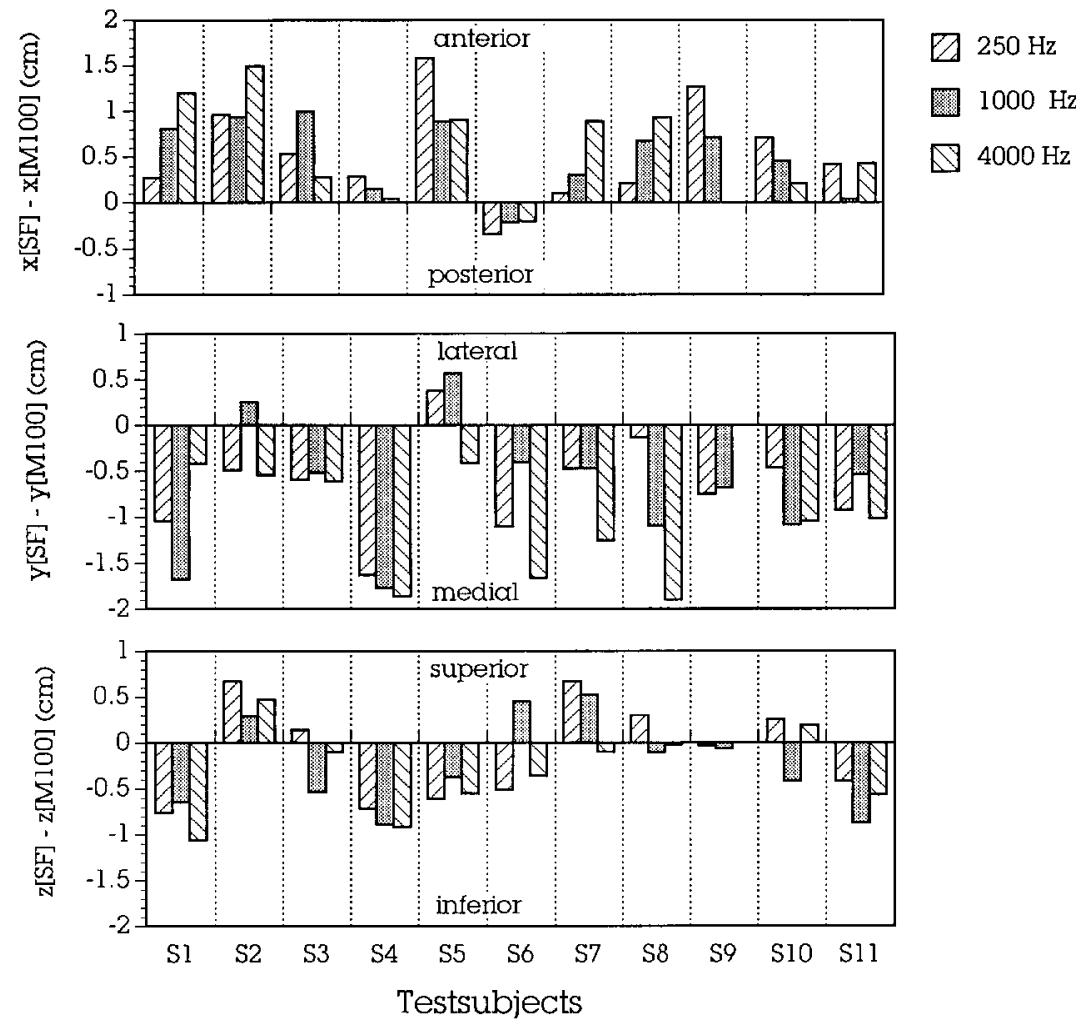

Fig. 2. Differences between the estimated source locations $(\mathrm{x}, \mathrm{y}, \mathrm{z})$ of the SF relative to M100 for each subject and each frequency investigated. The differences are presented in terms of the postero-anterior, medio-lateral and infero-superior dimensions of the head frame. The 3 frequencies used $(250,1000$, and $4000 \mathrm{~Hz})$ are indicated by different fill patterns. 
isocontour lines, but also a difference in location of the underlying current sources. It appears from these plots that the equivalent source of the SF is located more anterior and deeper relative to the M100 source.

Using the source localization procedure and the evaluation criteria described in Methods, the equivalent source locations for SF and M100 were determined for each stimulus frequency. The equivalent current dipole location of the M100 for each subject at each frequency can be used as an internal marker of the location of the subjects auditory cortex (Pantev 1992). Therefore these displays become largely independent of the individual neuroanatomy. The differences between the estimated source locations of the SF relative to the $\mathrm{M} 100$ are presented for each subject and separately for every frequency in Fig. 2 for the postero-anterior, medio-lateral and infero-superior directions of the head frame.

Fig. 2 demonstrates consistent differences between the SF and M100 locations in antero-posterior and medio-lateral directions. The MANOVA results confirm that, over the whole frequency range, the equivalent sources of the SF are located more anterior - about $6 \mathrm{~mm}(F(1,9)=15.7, P<0.01)$ and more medial - about $9 \mathrm{~mm}(F(1,9)=23.45, P<0.001)$ than the corresponding equivalent sources of the M100.

Figs. 3 and 4 show the dependence of the estimated distance of the source location of the M100 and the SF from the origin of the 3-dimensional (3-D) Cartesian coordinate system in the medio-lateral direction for each test subject investigated. This value represents indirectly the depth of the corresponding equivalent source. Fig. 3 shows on a semi-logarithmic scale the results of M100 obtained for each subject.

The absolute values on the y-axes of the 11 scatterplots may vary across subjects. The difference between the highest and the lowest values, however, amounts to $3 \mathrm{~cm}$ in all subjects.

In scatterplots for which tonotopic organization for the source locations was obvious, filled circles were used. In 2 out of the 11 subjects (S5 and S8) tonotopicity of the supratemporal cortex remained obscure (filled squares); in one other case (S9), the tonotopic representation was weak. The thick lines inserted into the scatterplots represent logarithmic fits. The coefficients of determination (squared correlation coefficients) of

\section{M100}
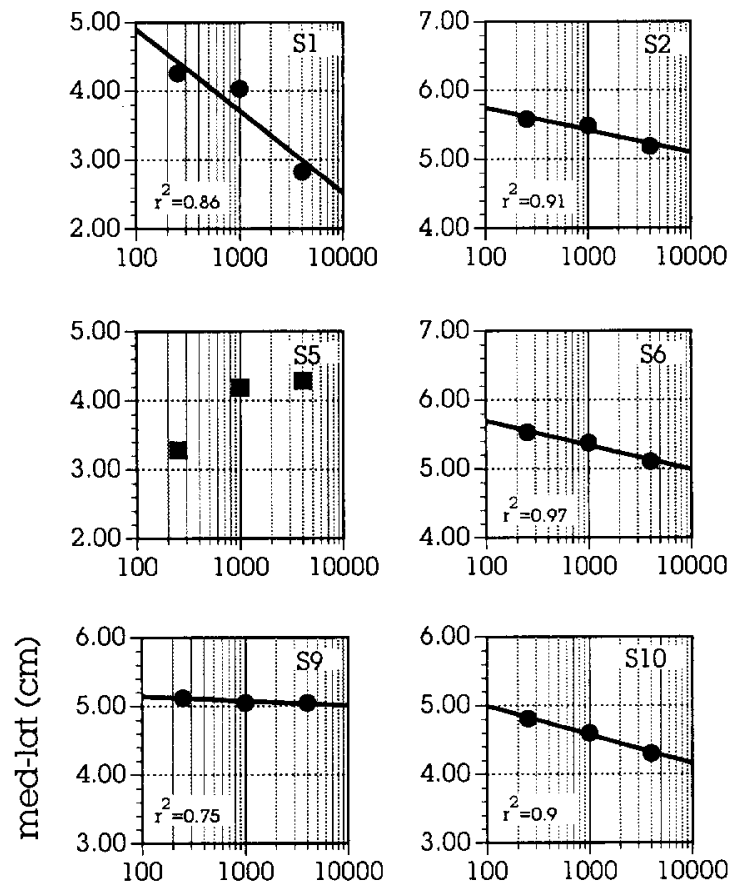
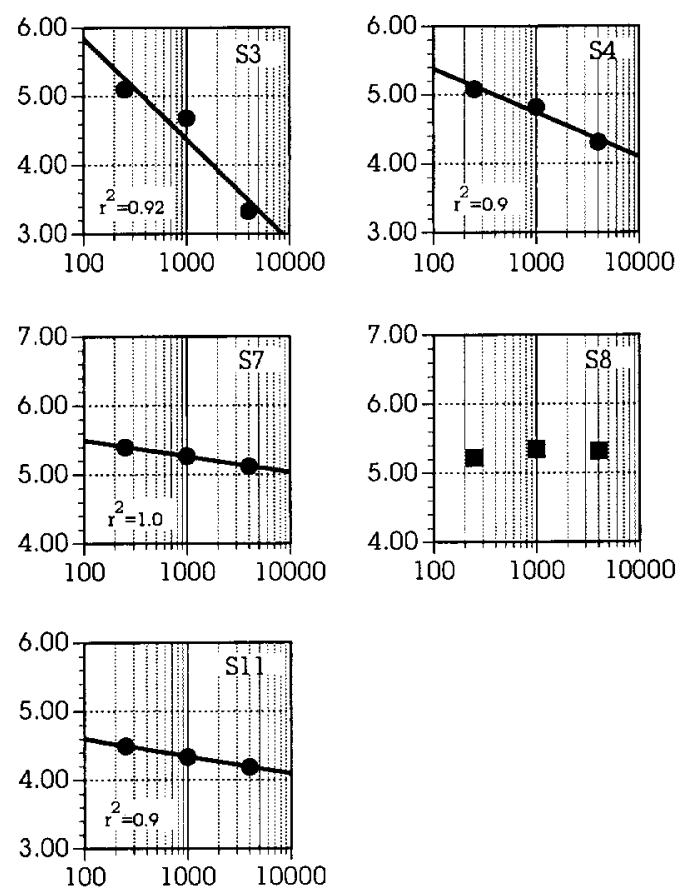

\section{frequency $(\mathrm{Hz})$}

Fig. 3. Dependence of the estimated distance of the source location of the M100 from the origin of the coordinate system of the head frame in the medio-lateral direction for each subject investigated. This value represents indirectly the depth of the corresponding equivalent source and is shown on semi-logarithmic scale. The abscissa represents the stimulus frequency, and the ordinate the y coordinate of the estimated source location. Although the values on the y-axes vary across subjects, the difference between the highest and the lowest values amounts to a constant 3 $\mathrm{cm}$. The thick lines represent logarithmic function fits to the y values. The coefficients of determination (squared correlation coefficients) of these functions are noted in the lower left part of each plot. 
$\mathrm{SF}$
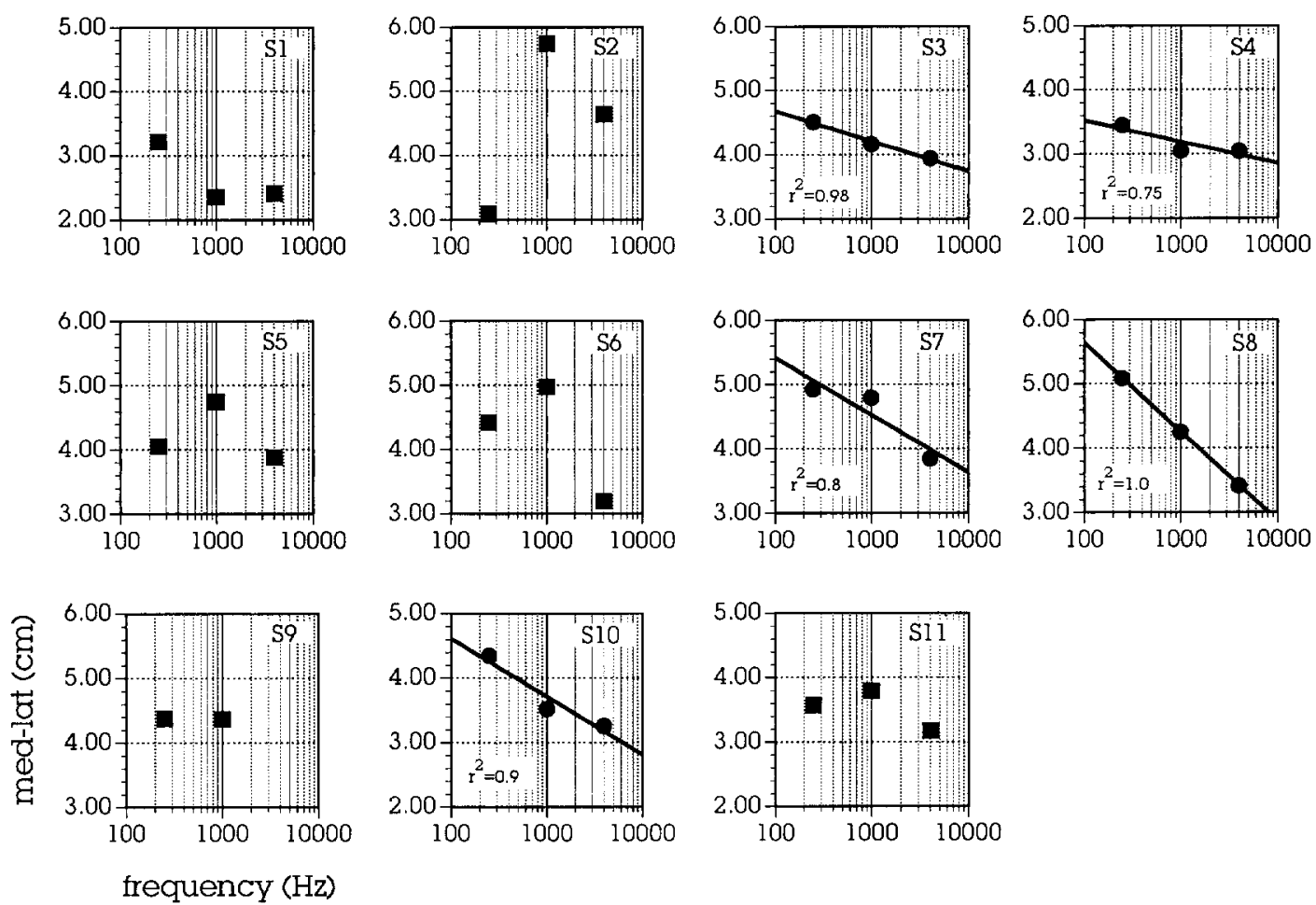

Fig. 4. Same as Fig. 3, but for the SF.

these functions are noted in the lower left part of each scatterplot. The calculated mean coefficient of determination amounts to a value of 0.95 .

The corresponding results obtained for the equivalent sources of the sustained field are illustrated in Fig. 4.

Compared to the M100 data, the tonotopic organization of the source locations for the 3 stimulus frequencies is less evident in the SF data. The effect, however, can be seen in 5 out of the 11 subjects. In 4 cases where a tonotopic representation is obvious in the M100 data (S1, S2, S6 and S11) it cannot be detected in the SF data. The main reason for this failure is that the value of the $y$ coordinate of the source location for the $250 \mathrm{~Hz}$ stimulus frequency is smaller than the respective value at $1000 \mathrm{~Hz}$. In subject S9, the dipole fit calculations for the $4000 \mathrm{~Hz}$ stimulus frequency failed. In subject S5, neither the M100 nor the SF data showed a tonotopic organization for equivalent cortical sources corresponding to different stimulus frequencies. In S8, for whom no signs of M100 tonotopicity are present, a clear tonotopic representation is shown for the SF with a coefficient of determination of 1 . Regression analysis on y coordinates of the source locations of the M100 and SF over the frequency range between 250 and $4000 \mathrm{~Hz}$ showed similar slope coefficients of the corresponding regression lines of -4 and $-5 \mathrm{~mm}$, respectively.
Finally, the individual $x, y$ and $z$ coordinates of the estimated source locations of the M100 and SF for the stimulus frequencies 250,1000 and $4000 \mathrm{~Hz}$ were averaged across all subjects. These averaged values, presented as a 3-D plot in Fig. 5, are intended to illustrate "centres of activity" of the excited neuronal popula-

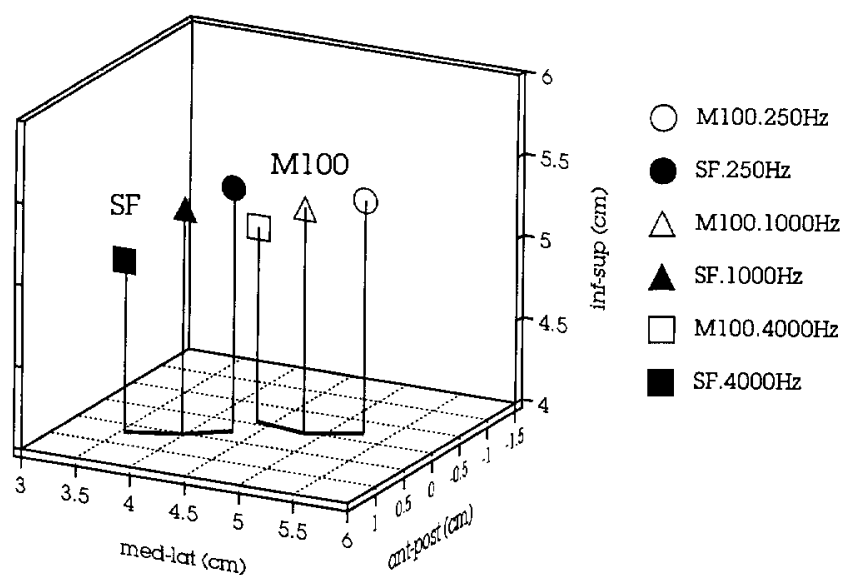

Fig. 5. Mean estimated source locations of the M100 and the SF for the stimulus frequencies 250,1000 and $4000 \mathrm{~Hz}$. The empty symbols represent the averaged source locations of the M100; filled symbols correspond to the averaged source locations of the SF. The 3 stimulus frequencies are coded with different symbols. Antero-posterior, medio-lateral and infero-superior axes of the 3-D plot refer to the head-based coordinate system described in the Methods section of the text. 
tions generating the two evoked responses: the M100 of the slow AEF, and the SF.

Statistical evaluation of the data (MANOVA, $F(1$, $9)=17.5, P<0.01)$ confirmed the visual impression that there is a significant difference between the source locations of the M100 and the SF.

\section{Discussion}

\section{Differences between the origins of $S F$ and $M 100$}

The auditory evoked sustained response reflects an important aspect of central auditory processing, indicating cortical activity that occurs throughout the sensation of the sound rather than in response to its onset or offset. Unfortunately, there is limited information about the origin of the sustained response. The nature of this evoked response, as well as its underlying brain functions, are poorly understood. It may derive from both the primary auditory areas and the frontal association areas (Peronnet and Michel 1977). The apparent activity of these two regions during the appearance of the sustained potential indicates the functioning of extensive connections between temporal association areas and prefrontal regions of the brain. Although Picton et al. (1978c) found the maxima of the sustained potentials at the vertex and at frontal head regions, Hari et al. (1980) did not observe any magnetic SF above the fronto-central midline. This can easily be explained by a configuration of the sources of the sustained response being predominantly tangentially oriented in the supratemporal cortex, but radially oriented in the frontal association areas and thus invisible in magnetic records. The results of Picton et al. (1978c,d) showed that the SP extended less posteriorly than the N100 component of the transient evoked potential. More obvious are the source analysis results of the N100 and the SP reported by Scherg et al. (1989) and Scherg and Picton (1991). These authors found the source of the SP to be located anteriorly to the N100 source. Another major distinction between the sources of the N100 and the SP was their different orientations, SP being inclined more anteriorly and medially. These findings are in line with both the results of the neuromagnetic study reported by Hari et al. (1987a,b) and with the present results, in which the source of the SF was found to be located significantly more anterior than the source of the M100. We found an average difference between the two source locations of about 6 $\mathrm{mm}$ in the antero-posterior direction, in line with the observation of Hari et al. (1987). Additionally, the source of the SF was found to be located $9 \mathrm{~mm}$ more medial than the corresponding source of the M100.

\section{Tonotopic organization of the human auditory cortex}

The most characteristic features of the tonotopic representation of stimulus frequency in the location of the equivalent source of the M100 component in the supratemporal cortex are that lower frequencies are usually located more superficially than higher ones, and that the depth of the M100 equivalent source increases linearly with the logarithm of the stimulus frequency (Romani et al. 1982; Pantev et al. 1988; Yamamoto et al. 1988). In this comparative study between the source locations of the SF and slow AEF tonotopicity was checked for a larger group of normally hearing subjects. Tonotopicity was better seen in the behaviour of the M100 source than in that of the SF. In most of the subjects in whom the tonotopic representation of the magnetic M100 and SF sources could not be seen, the reason was mainly the behaviour of the equivalent source for the $250 \mathrm{~Hz}$ stimulus, which was located more superficially than the source for 1000 Hz. Although we are not able to explain this result satisfactorily, a probable explanation may be found in the differences of individual neuroanatomy. The individual neuroanatomy may cause such local orientation of the cortical surface embedding the equivalent neuronal sources for $250 \mathrm{~Hz}$ that the cortical activity cannot be properly detected by the magnetic sensors, which are mainly sensitive to sources oriented tangentially to the head surface. Likewise, activity arising from the presumed second fronto-central generator may distort the computed source locations, depending on the individual neuroanatomy, assuming that it may include tangentialy oriented current sources in the frontal lobe.

Results of the regression analysis for the y coordinate of the M100 and of the SF source locations show that the depth of their equivalent current sources increases by about 2-3 $\mathrm{mm}$ when stimulus frequency increases by one octave. This confirms previous results obtained by Elberling et al. (1982), Romani et al. (1982), Pantev et al. (1988) and Yamamoto et al. (1988), and it seems to be a reasonable value compared to the size of the area of the primary auditory cortex and integration region, which have a combined volume estimated to be approximately $2.5-3.5 \mathrm{~cm}^{3}$ (Talairach and Tournoux 1988).

The tonotopic organization of the auditory cortex along isofrequency strips comprising frequency specific neurones is well known from animal experiments (Schreiner 1991). The source locations of the M100 and the SF were obtained over a wide range of frequencies $(250$ and $4000 \mathrm{~Hz})$ and averaged across 11 subjects. They illustrate the "centres of activity" of the excited neuronal populations which generate both evoked responses. It appears that these "centres of activity", represented by the centres of the estimated equivalent current dipoles, lie on two different sheets of the cortical surface. Thus the idea of spatial organization of anatomical substrates in the auditory cortex, corresponding to different electrophysiological phe- 
nomena (Hari et al. 1980), is nicely supported by the results of this study.

This work was supported by grants from the Deutsche Forschungsgemeinschaft (Klinische Forschergruppe "Biomagnetismus und Biosignalanalyse").

The authors thank Drs. R. Hari, T.W. Picton and S. Makeig for helpful comments.

\section{References}

Annett, M. The binomial distribution of right, mixed and left-handed. Quart. J. Exp. Psychol., 1967, 19: 327-333.

Bertrand, O., Perrin, P, and Pernier, J. Evidence for a tonotopic organisation of the auditory cortex observed with auditory evoked potentials. Acta Otolaryngol. (Stockh.), 1991, Suppl. 491: 116-123.

Butler, R.A. The effect of changes in stimulus frequency and intensity on habituation of the human vertex potential. J. Acoust. Soc. Am., 1968, 44: 945-950.

David, E., Finkenzeller, P., Kallert, S. and Keidel, W.D. Akustischen Reizen zugeordnete Gleichspannungsänderungen am intakten Schädel des Menschen. Pflügers Arch. Ges. Physiol., 1969, 309: 362-367.

Elberling, C., Bak, C., Kofoed, B., Lebech, J. and Saermark, K. Auditory magnetic fields from the human cortex, influence of stimulus intensity. Scand. Audiol., 1981, 10: 203-207.

Elberling, C., Bak, C., Kofoed, B., Lebech, J, and Saermark, K. Auditory magnetic fields. Source localization and "tonotopical organization" in the right hemisphere of the human brain. Scand. Audiol., 1982, 11: 61-65.

Elbert, T., Rockstroh, B., Hampson, S., Pantev, C. and Hoke, M. The magnetic counterpart of the contingent negative variation. Electroenceph. clin. Neurophysiol., 1993, in press.

Hari, R. Sensory Evoked Sustained Potentials in Man. Thesis for the degree of the Doctor of Medical Sciences, University of Helsinki, 1980.

Hari, R., Sams, M. and Järvilehto, T. Auditory evoked transient sustained potentials in the human EEG.I. Effects of expectation of stimuli. Psychiat. Res., 1979a, 1: 297-306.

Hari, R., Sams, M. and Järvilehto, T. Auditory evoked transient sustained potentials in the human EEG. II. Effects of small doses of ethanol. Psychiat. Res., 1979b, 1: 307-312.

Hari, R., Aittoniemi, M., Jarvinen, M.L., Katila, T. and Varpula, T. Auditory evoked transient and sustained magnetic fields of the human brain. Exp. Brain Res., 1980, 40: 237-240.

Hari, R., Pelizzone, M., Mäkelä, J.P., Hallstroem, J., Leinionen, L. and Lounasmaa, O.V. Neuromagnetic responses of the human auditory cortex to on- and offsets of noise bursts. Audiology, 1987, 26: 31-43.

Hoke, M. Auditory evoked magnetic fields. In: E. Başar (Ed.), Dynamics of Cognitive and Sensory Processing by the Brain. Springer, Berlin, 1988: 311-318.

Järvilehto, T. and Fruhstorfer, H. Is the sound-evoked DC potential a contingent negative variation? Electroenceph. clin. Neurophysiol., 1973, Suppl. 33: 105-108.

Keidel, W.D.D.C.-potentials in the auditory evoked response in man. Acta Otolaryngol. (Stockh.), 1971, 71: 242-248.

Köhler, W. and Wegener, J. Currents of the human auditory cortex. J. Cell. Comp. Physiol., 1955, 45: 25-54.

Köhler, W., Held, R. and O'Connell, D. An investigation of cortical currents. Proc. Am. Phil. Soc., 1952, 96: 290-330.

Lauter, J.L., Herschovitch, P., Formby, C. and Raichle, M.E. Tonotopic organization in the human auditory cortex revealed by positron emission tomography. Hearing Res., 1985, 20: 199-205.
Merzenich, M.M. and Brugge, J.F. Representation of the cochlear position on the superior temporal plane of the macaque monkey. Brain Res., 1973, 50: 275-296.

Merzenich, M.M., Knight, P.L. and Roth, G.L. Representation of cochlea within primary auditory cortex in cat. J. Neurophysiol., 1975, 38: 231-249.

Merzenich, M.M., Kaas, J.H. and Roth, G.L. Comparison of tonotopic maps in animals. J. Comp. Neurol., 1976, 166: 387-402.

Pantev, C. Neuromagnetic source localization using a large array biomagnetometer. Biomed. Res., 1992, Suppl. 1: 11-16.

Pantev, C., Lütkenhöner, B., Hoke, M. and Lehnertz, K. Comparison between simultaneously recorded auditory-evoked magnetic fields and potentials elicited by ipsilateral, contralateral and binaural tone burst stimulation. Audiology, 1986, 25: 54-61.

Pantev, C., Hoke, M., Lehnertz, K., Lütkenhöner, B., Anogianakis, G. and Wittkowski, W. Tonotopic organization of the human auditory cortex revealed by transient auditory evoked magnetic fields. Electroenceph. clin. Neurophysiol., 1988, 69: 160-170.

Pantev, C., Hoke, M., Lütkenhöner, B. and Lehnertz, K. Tonotopic organization of the auditory cortex: pitch versus frequency representation. Science, 1989, 246: 486-488.

Pantev, C., Hoke, M., Lehnertz, K., Lütkenhöner, B., Fahrendorf, G. and Stöber, U. Identification of sources of brain neuronal activity with high spatiotemporal resolution through combination of neuromagnetic source localization (NMSL) and magnetic resonance imaging (MRI). Electroenceph. clin. Neurophysiol., 1990, 75: 173-184.

Pantev, C., Hoke, M., Lütkenhöner, B. and Lehnertz, K. Neuromagnetic evidence of functional organization of the auditory cortex in humans. Acta Otolaryngol. (Stockh.), 1991a, Suppl. 491: 106-115.

Pantev, C., Gallen, C., Hampson, S., Buchanan, S. and Sobel, D. Reproducibility and validity of neuromagnetic source localization using a large array biomagnetometer. Am. J. EEG Technol., 1991b, 31: 83-101.

Papanicolaou, A.C., Baumann, S., Rogers, R.L., Saydjari, C., Amparo, E.G. and Eisenberg, H.M. Localization of auditory response sources using magnetoencephalography and magnetic resonance imaging. Clin. Neurophysiol., 1990, 47: 33-37.

Peronnet, F. and Michel, F. The Asymmetry of the Auditory Potentials in Normal Man and in Patients with Brain Lesions, Vol. 2. Karger, Basel, 1977: 130-141.

Picton, T.W., Hillyard, S.A., Krausz, H.I. and Galambos, R. Human auditory evoked potentials. I. Evaluation of components. Electroenceph. clin. Neurophysiol., 1974, 36: 179-190.

Picton, T.W., Woods, D.L., Baribeau-Braun, J. and Healey, T.M.G. Evoked potential audiometry. J. Otolaryngol., 1977, 6: 90-118.

Picton, T.W., Woods, D.L. and Proulx, G.B. Human auditory sustained potentials. I. The nature of the response. Electroenceph. clin. Neurophysiol., 1978a, 45: 186-197.

Picton, T.W., Woods, D.L. and Proulx, G.B. Human auditory sustained potentials. II. Stimulus relationships. Electroenceph. clin. Neurophysiol., 1978b, 45: 198-210.

Picton, T.W., Campbell, K.B., Baribeau-Braun, J. and Proulx, G.B. The neurophysiology of human attention: a tutorial review. In: J. Requin (Ed.), Attention and Performance, VII. Lawrence Erlbaum, Hillsdale, NJ, 1978c: 429-467.

Picton, T.W., Woods, D., Stuss, D. and Campbell, K. Methodology and meaning of human evoked potential scalp distribution studies. In: D. Otto (Ed.), Multi-disciplinary Perspectives in EventRelated Brain Potential Research. US Gov. Printing Office, Washington, DC, 1978d: 515-522.

Reite, M., Zimmerman, J.T. and Zimmerman, J.E. Magnetic auditory evoked fields: interhemispheric asymmetry. Electroenceph. clin. Neurophysiol., 1981, 51: 388-392.

Romani, G.L., Williamson, S.J. and Kaufman, L. Tonotopic organization of the human auditory cortex. Science, 1982, 216: 13391340 . 
Sarvas, J. Basic mathematical and electromagnetic concepts of the biomagnetic inverse problem. Phys. Med. Biol., 1987, 32: 11-22.

Scherg, M., Vajsar, J. and Picton, T.W. A source analysis of the late human auditory evoked potentials. J. Cogn. Neurosci., 1989, 4: 336-354.

Scherg, M. and Picton, T.W. Separation and identification of eventrelated potential components by brain electric source analysis. In: C.H.M. Brunia, G. Mulder and M.N. Verbaten (Eds.), Event-Related Brain Research. EEG Suppl. 42. Elsevier, Amsterdam, 1991: 24-37.
Schreiner, C.E. Functional topographies in the primary auditory cortex of the cat. Acta Otolaryngol. (Stockh.), 1991, Suppl. 491: 7-16.

Talairach, J. and Tournoux, P. Co-Planar Stereotaxic Atlas of the Human Brain. Thieme Verlag, Stuttgart, 1988.

Yamamoto, T., Williamson, S.J., Kaufman, L., Nicholson, C. and Llinás, R. Magnetic localization of neural activity in the human brain. Proc. Nat. Acad. Sci. (USA), 1988, 85: 8732-8736. 